\title{
Performance analysis of MIMO-SESS with Alamouti scheme over Rayleigh fading channels
}

\author{
Shichuan Ma*, Lim Nguyen, Yaoqing (Lamar) Yang and Won Mee Jang
}

\begin{abstract}
Self-encoded spread spectrum (SESS) is a novel modulation technique that acquires its spreading sequence from the random input data stream rather than through the use of the traditional pseudo-noise code generator. It has been incorporated with multiple-input multiple-output (MIMO) systems as a means to combat fading in wireless channels. In this paper, we present the analytical study of the bit-error rate (BER) performance of MIMO-SESS systems under Rayleigh fading. The BER expressions are derived in closed form, and the veracity of the analysis is confirmed by numerical calculations that demonstrate excellent agreement with simulation results. The performance analysis shows that the effects of fading can be effectively mitigated by taking advantage of spatial and temporal diversities. For example, a $2 \times 2$ MIMO-SESS system can achieve about $7 \mathrm{~dB}$ performance improvement at $10^{-4} \mathrm{BER}$ over a MIMO PN-coded spread spectrum system.
\end{abstract}

Keywords: self-encoded spread spectrum, iterative detection, multiple-input multiple-output, Alamouti scheme

\section{Introduction}

Conventional direct sequence spread spectrum system employs pseudo-noise (PN) code generators which are typically linear feedback shift register circuits that generate maximal length or related sequences. In contrast, by deriving its spreading sequences from the user data stream and obviating the need for PN code generators, self-encoded spread spectrum (SESS) provides a feasible implementation of random-coded spread spectrum and has a number of unique features such as the potential for enhanced transmission security and anti-jamming capability, multi-rate applications, modulation gain and inherent time diversity [1-7]. The modulation memory associated with self-encoding was exploited by iterative detection to achieve a significant gain [6].

Recently, SESS was incorporated with multiple-input multiple-output (MIMO) Alamouti scheme to further improve the performance [8]. The spatial diversity from multiple antennas at the transmitter (TX) and receiver (RX) enables MIMO techniques to be effective against channel fading [9-11] and the technology has been standardized [12-14]. In a recent report [15], we have extended our work in [8] to orthogonal space-time

\footnotetext{
* Correspondence: sma@huskers.unl.edu

Department of Computer and Electronics Engineering, University of Nebraska-Lincoln, Omaha, NE 68182, USA
}

block codes with multiple iterations and have shown via simulation that MIMO-SESS provides a novel means to combat fading in wireless channels by exploiting diversities in both space and time domains. However, the theoretical analysis of MIMO-SESS in Rayleigh fading channels has not been conducted.

In this paper, we thus extend our previous work in [8] to present the analytical study of the BER performance of SESS with MIMO-Alamouti scheme under Rayleigh fading. We first derived BER of the correlation detector in closed form and verified the performance improvement due to spatial diversity. Next, we derived the closed-form BER expression of the time-diversity SESS detector that further improves the performance from the combined spatial and temporal diversities. We then investigate the performance of the iterative detector that combines the outputs of the correlation and time-diversity detectors. By approximating the probability density function (pdf) associated with the time-diversity detector by a Dirac delta function, we obtained a lower-bound expression for the BER of the iterative detector and verified with simulations that the bound is tight even for relatively small spreading lengths.

This paper is organized as follows. MIMO-SESS system with iterative detection is described in the next section. Section 3 presents the theoretical analysis of the 
system. The BER performance of the correlation and diversity detectors is analyzed in closed form, and a lower bound for the iterative detector is derived. Section 4 presents the numerical results to validate the analysis, and Section 5 concludes the paper.

Notations: The superscripts " and $T$ represent the conjugate and the transpose operations, respectively. $|\cdot|$, $\mathcal{E}\{\cdot\}$, and $\operatorname{diag}(\cdot)$ denote the absolute value of a scalar, the expectation operation, and the diagonal vector of a matrix, respectively. Matrix (vector) is represented by capital (small) bold letter. The Q-function, gamma function, and incomplete gamma function are denoted by $Q$ $(x), \Gamma(a)$, and $\Gamma(a, x)$, respectively. Dirac delta function is represented by $\delta(\cdot)$.

\section{System model}

In this section, the transceiver of the MIMO-SESS system with iterative detection is described.

\subsection{MIMO-SESS transmitter}

The block diagram of the proposed transmitter is shown in Figure 1, where the rounded corner blocks represent $N$ delay registers and $T_{b}$ is the bit interval. Alamouti scheme-based space-time block coding (STBC) is utilized to achieve transmit diversity.
The source information $b$ is assumed to be bi-polar values of $\pm \sqrt{E_{b} / 2}$, where $E_{b}$ is the average transmit energy per bit. It should be mentioned that the energy per transmit antenna is one half of the total transmit energy in order to make the multi-antenna system comparable to a single antenna system. The bits are first spread by the self-encoded spreading sequence of length $N$ at a chip rate of $N / T_{b}$. This sequence is constructed from the user's information stored in the delay registers that are updated every $T_{b}$. Thus, with a random input data stream, the sequence is also random and time-varying from one bit to another. For example, the spreading sequence for the $k$ th bit, $b(k)$, is given as

$$
\mathbf{s}(k)=[b(k-1) b(k-2), \ldots, b(k-N)]^{T},
$$

and the spreading chips are given as

$$
\mathbf{c}(k=b(k) \mathbf{s}(k) \text {. }
$$

To facilitate the description in the sequel, let $c(k, n)$ denote the $n$th chip of the $k$ th bit, which can be expressed as

$$
c(k, n)=b(k) b(k-n) .
$$

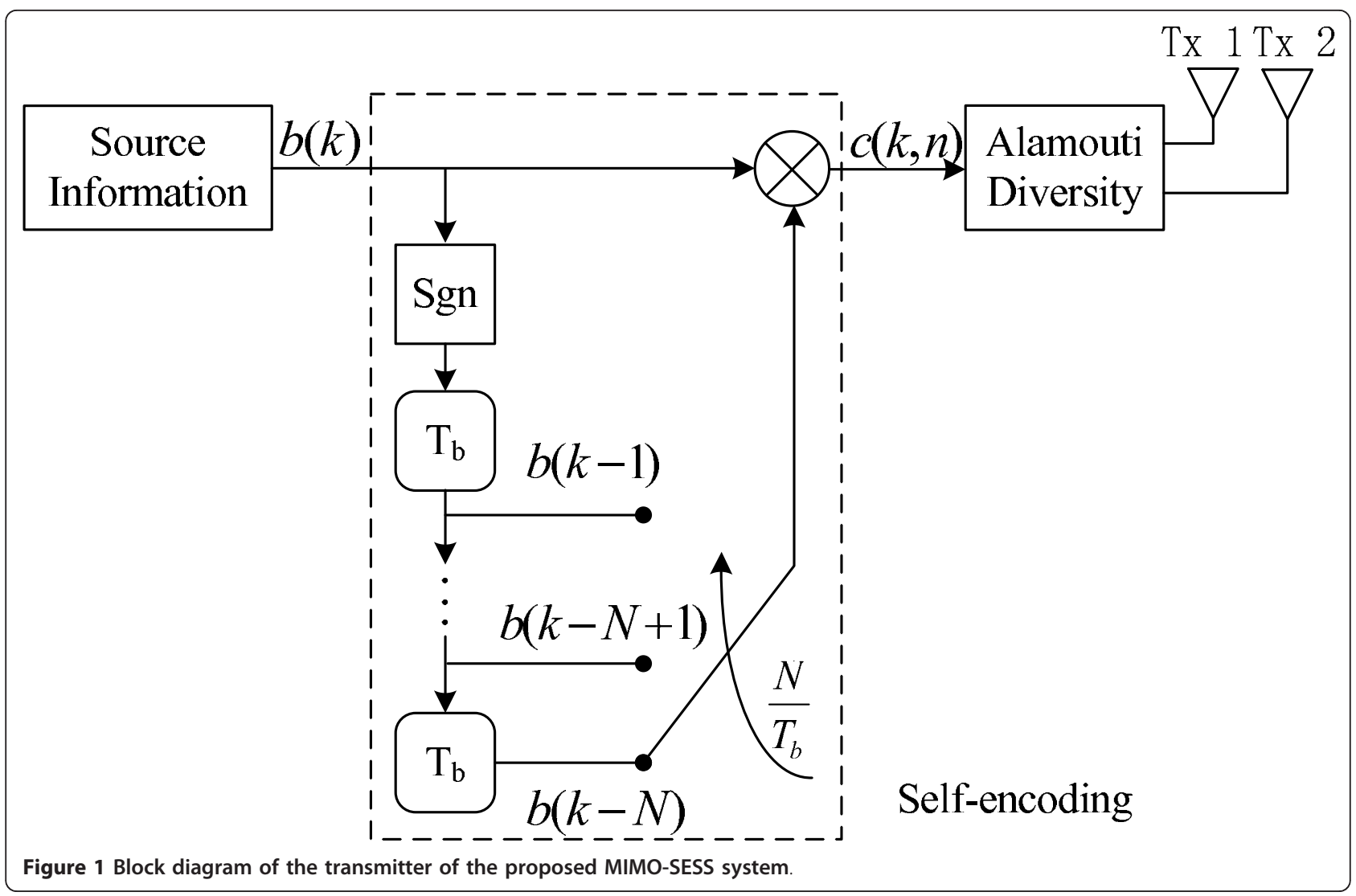


The spreading chips are then divided into two streams by applying Alam-outi scheme, where a block of two consecutive chips, $c(k, 2 i)$ and $c(k, 2 i+1)$, are transmitted by sending $c(k, 2 i),-c^{*}(k, 2 i+1)$ to the first antenna, and $c(k, 2 i+1), c^{*}(k, 2 i)$ to the second antenna. Here, $i \in\{0,1, \ldots, N / 2-1\}$ is the block index for the chips of the $k$ th bit. The signals are then transmitted over a MIMO fading channel.

The channel between each transmit and receive antenna pair is assumed to undergo Rayleigh fading that remains constant over $T_{b}$ but is independent from bit to bit $[6,16]$. The channels for different transmit/receive antenna pair are independent.

\subsection{MIMO-SESS receiver}

Figure 2 shows the block diagram of the receiver. For the $m$ th antenna, the received signals within the $i$ th block are given as

$$
\begin{aligned}
& x_{m}(k, 2 i)=h_{1, m}(k) c(k, 2 i)+h_{2, m}(k) c(k, 2 i+1)+e_{m}(k, 2 i),(4) \\
& x_{m}(k, 2 i+1)=-h_{1, m}(k) c^{*}(k, 2 i+1)+h_{2, m}(k) c^{*}(k, 2 i)+e_{m}(k, 2 i+1),
\end{aligned}
$$

where $h_{1, m}(k)$ and $h_{2, m}(k)$ respectively denote the normalized complex channel impulse response coefficients for the $k$ th bit between the 1 st and $2^{\text {nd }}$ transmit antennas and the $m$ th receive antenna; $e_{m}$ is a Gaussian noise with zero mean and variance $N N_{0} / 2$. It should be noted that the noise is broadband because it is sampled at chip level. Its variance is thus the narrow-band noise variance $N_{0} / 2$ multiplied by the spreading factor $N$.

\subsubsection{Diversity combining}

We assume that the delay registers in the receiver have been synchronized with the transmitter [7], and that perfect channel knowledge is available at the receiver. Under these assumptions, diversity combining from the $M$ receiver antennas is carried out over two consecutive chip intervals according to $[9,17]$ as

$$
\begin{aligned}
& \begin{aligned}
y(k, 2 i) & =\sum_{m=1}^{M}\left(h_{1, m}^{*}(k) x_{m}(k, 2 i)+h_{2, m}(k) x_{m}^{*}(k, 2 i+1)\right)+w(k, 2 i) \\
& =\alpha(k) c(k, 2 i)+w(k, 2 i),
\end{aligned} \\
& y(k, 2 i+1)=\sum_{m=1}^{M}\left(h_{2, m}^{*}(k) x_{m}(k, 2 i)-h_{1, m}(k) x_{m}^{*}(k, 2 i+1)\right)+w(k, 2 i+1) \\
& =\alpha(k) c(k, 2 i+1)+w(k, 2 i+1),
\end{aligned}
$$

where

$$
\alpha(k)=\sum_{m=1}^{M}\left(\left|h_{1, m}(k)\right|^{2}+\left|h_{2, m}(k)\right|^{2}\right)
$$

and

$$
w(k, n)=\left\{\begin{array}{l}
\sum_{m=1}^{M}\left(h_{1, m}^{*}(k) e_{m}(k, n)+h_{2, m}(k) e_{m}^{*}(k, n+1)\right), \text { if } n \text { is even } \\
\sum_{m=1}^{M}\left(h_{2, m}^{*}(k) e_{m}(k, n-1)-h_{1, m}(k) e_{m}^{*}(k, n)\right), \text { if } n \text { is odd }
\end{array}\right.
$$

Because the channel coefficients and the white noise are independent, the noise term $w$ is a random variable with zero means and variance $M N N_{0}$.

The combined signals given in (6) and (7) can be written in one equation as

$$
\gamma(k, n)=\alpha(k) c(k, n)+w(k, n),
$$

and further in vector form as

$$
\mathbf{y}(k)=\alpha(k) \mathbf{c}(k)+\mathbf{w}(k),
$$

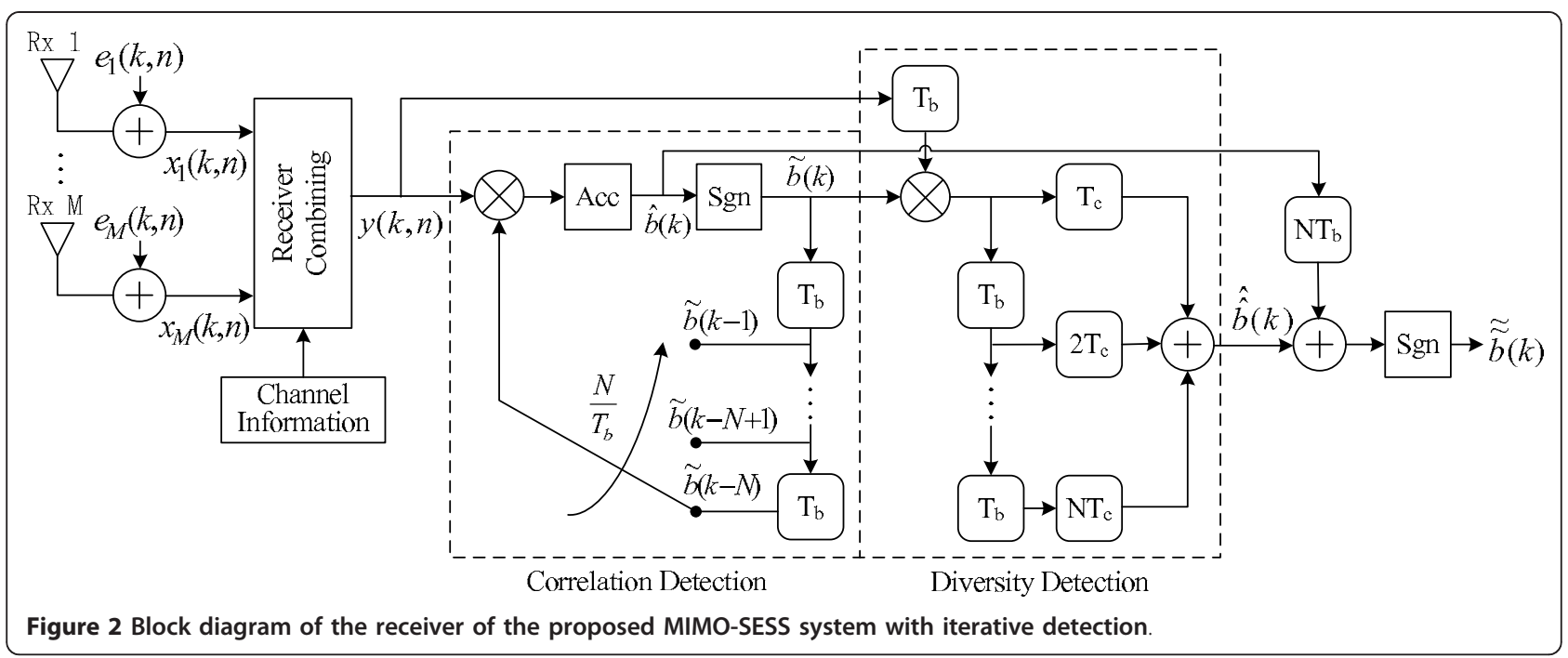


where

$$
\mathbf{y}(k)=[\gamma(k, 1) y(k, 2), \ldots, y(k, N)]^{T},
$$

and

$$
\mathbf{w}(k)=[w(k, 1) w(k, 2), \ldots, w(k, N)]^{T} .
$$

\subsubsection{Correlation detection}

After diversity combining, the chips are despread and detected. The correlation estimate of the $k$ th bit is given as

$$
\begin{aligned}
\hat{b}(k) & =\frac{1}{N} \mathbf{y}^{T}(k) \mathbf{s}(k) \\
& =\alpha(k) b(k)+v_{1}(k)
\end{aligned}
$$

where

$$
v_{1}(k)=\frac{1}{N} \sum_{n=1}^{N} w(k, n) b(k-n)
$$

is a random variable with zero mean and variance $M N_{0}$. The conditional SNR of the correlation detection given the channel coefficients is

$$
\gamma_{1}=\frac{|\alpha(k) b(k)|^{2}}{\mathcal{E}\left\{\left|v_{1}(k)\right|^{2}\right\}} .
$$

Because the channel fading coefficients, the information bits, and the thermal noise are independent of each other, it is easy to see that Equation (16) is reduced to

$$
\gamma_{1}=\frac{E_{b}}{N_{0}} \alpha(k)
$$

A hard decision is then performed on the correlation output, which in turn is fed back to the receiver delay registers in order to update the dispreading sequence for the next bit.

$$
r C i \tilde{b}(k)=\operatorname{sgn}[\hat{b}(k)]=\left\{\begin{aligned}
1, & \hat{b}(k)>0 \\
-1, & \hat{b}(k)<0
\end{aligned}\right.
$$

This also provides an estimate of the corresponding bit if no iterative detection is employed.

\subsubsection{Time-diversity detection}

SESS provides a unique encoding scheme that can be utilized to achieve temporal diversity. To better illustrate this mechanism, let us write the following $N^{2}$ chips in a square matrix as

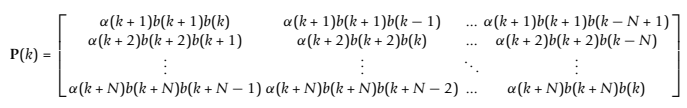

where each element represents a chip as given in (10) and each row includes the chips of one bit. For simplicity, the noise term is omitted.

We observe that the $k$ th bit, $b(k)$, is present in the diagonal elements of the matrix $\mathbf{P}(k)$. This means that the information of the $k$ th bit is effectively transmitted in the next $N$ bits, which is a unique characteristic of the SESS modulation scheme. By defining $\mathbf{d}(k)=\operatorname{diag}(\mathbf{P}$ $(k))$, the diversity estimate of $b(k)$ can be obtained from the hard decisions of the next $N$ bits, $[\tilde{b}(k+1) \tilde{b}(k+2), \ldots, \tilde{b}(k+N)]$, as

$$
\begin{aligned}
\widehat{\hat{b}}(k) & =\frac{1}{N} \mathbf{d}(k)[\tilde{b}(k+1) \tilde{b}(k+2), \ldots, \tilde{b}(k+N)]^{T}+v_{2}(k) \\
& =\frac{1}{N} \sum_{n=1}^{N} \alpha(k+n) b(k)+v_{2}(k),
\end{aligned}
$$

where

$$
v_{2}(k)=\frac{1}{N} \sum_{n=1}^{N} w(k+n, n) \tilde{b}(k+n)
$$

is a random variable with zero mean and variance $M N_{0}$. Notice that the effect of the possible bit errors from the correlation detection has been ignored - this is justified with sufficient SNR, as shown in Section 4.

The conditional SNR, given the channel coefficients for the diversity detection, is

$$
\gamma_{2}=\frac{\left|\frac{1}{N} \sum_{n=1}^{N} \alpha(k+n) b(k)\right|^{2}}{\mathcal{E}\left\{\left|v_{2}(k)\right|^{2}\right\}} .
$$

Because $\alpha(k+n), n \in\{1,2, \ldots, N\}, b(k)$, and $v_{2}(k)$ are independent of each other, Equation (22) reduces to

$$
\gamma_{2}=\frac{E_{b}}{N N_{0}} \sum_{n=1}^{N} \alpha(k+n) .
$$

It should be noted that the diversity detection introduces a time delay of $N T_{b}$ and that the structure is similar to a Rake receiver employing $N$ fingers to exploit the temporal diversity of SESS signals.

\subsubsection{Iterative detection}

The estimate of $b(k)$ can be improved iteratively with the summation of the correlation estimate, $\hat{b}(k)$, and the diversity estimate, $\widehat{\hat{b}}(k)$. Thus, we define

$$
\begin{aligned}
\breve{b}(k) & =\hat{b}(k)+\widehat{\widehat{b}}(k) \\
& =\left(\alpha(k)+\frac{1}{N} \sum_{n=1}^{N} \alpha(k+n)\right) b(k)+v(k),
\end{aligned}
$$


where $v(k)=v_{1}(k)+v_{2}(k)$.

The conditional SNR of the iterative detection, conditioned on the channel coefficients, is the summation of the SNR of the two independent signals, as follows:

$$
\begin{aligned}
\gamma & =\frac{\left|\left(\alpha(k)+\frac{1}{N} \sum_{n=1}^{N} \alpha(k+n)\right) b(k)\right|^{2}}{\mathcal{E}\left\{|v(k)|^{2}\right\}} \\
& =\frac{E_{b}}{N_{0}}\left[\alpha(k)+\frac{1}{N} \sum_{n=1}^{N} \alpha(k+n)\right] \\
& =\gamma_{1}+\gamma_{2} .
\end{aligned}
$$

The final hard decision is then obtained by

$$
r C \widetilde{\tilde{l}}(k)=\operatorname{sgn}[\breve{b}(k)]=\left\{\begin{array}{r}
1, \breve{b}(k)>0 \\
-1, \breve{b}(k)<0
\end{array}\right.
$$

\section{Performance analysis}

In this section, we analyze the distribution of the SNRs given in (17), (23) and (25), and the corresponding BER expressions. We proceed with the distribution of the normalized channel gain, $|h(k)|^{2}$. It is well known that the coefficients of the Rayleigh fading channel is generated as $h(k)=\left(h_{r}+j h_{i}\right) / \sqrt{2}$, where $h_{r}$ and $h_{i}$ are real values and normally distributed as $\mathcal{N}(0,1)$. The denominator $\sqrt{2}$ is for power normalization and the channel gain $|h(k)|^{2}=(\mid$ $\left.\left.h_{r}\right|^{2}+\left|h_{i}\right|^{2}\right) / 2$, is then gamma-distributed as $\Gamma(1,1)[18]$.

Because the summation of independent and identically distributed (i.i.d.) gamma random variables still follows a gamma distribution [18], the spatial diversity gain $\alpha(k)$ given in $(8)$ has a $\Gamma(2 M, 1)$ distribution. Furthermore, the conditional SNR of the correlation detector given in (17) follows $\Gamma\left(2 M, E_{b} / N_{0}\right)$ distribution, i.e.,

$$
p_{\gamma_{1}}(\gamma)=\frac{\gamma^{2 M-1} e^{-\frac{\gamma}{E_{b} / N_{0}}}}{(2 M-1) !\left(E_{b} / N_{0}\right)^{2 M}}, \quad \gamma \geq 0 .
$$

Now the bit-error probability for binary phase shift keying (BPSK) in AWGN with an SNR of $E_{b} / N_{0}$ is given in [16] as

$$
P_{b}=Q\left(\sqrt{\frac{2 E_{b}}{N_{0}}}\right)
$$

Given the conditional SNR of $p_{\gamma_{1}}(\gamma)$, the BER expression for the correlation detection is given by [[16], Eq. 14.4-15]:

$$
\begin{aligned}
P_{e, \text { corr }} & =\int_{0}^{\infty} Q(\sqrt{2 \gamma}) p_{\gamma_{1}}(\gamma) \mathrm{d} \gamma \\
& =\left[\frac{1}{2}\left(1-\mu_{1}\right)\right]^{2 M} \sum_{i=0}^{2 M-1}\left(\begin{array}{c}
2 M-1+i \\
i
\end{array}\right)\left[\frac{1}{2}\left(1+\mu_{1}\right)\right]^{i}
\end{aligned}
$$

where

$$
\mu_{1} \triangleq \sqrt{\frac{E_{b} / N_{0}}{1+E_{b} / N_{0}}}
$$

A similar analysis shows that the conditional SNR of the time-diversity detector given in (23) follows a $\Gamma\left(2 N M, E_{b} / N_{0} / N\right)$ distribution, i.e.,

$$
p_{\gamma_{2}}(\gamma)=\frac{\gamma^{2 N M-1} e^{-\frac{\gamma}{E_{b} / N_{0} / N}}}{(2 N M-1) !\left(E_{b} / N_{0} / N\right)^{2 N M}}, \quad \gamma \geq 0 .
$$

and the BER expression for the diversity detection is then obtained as

$$
\begin{aligned}
P_{e, \mathrm{div}} & =\int_{0}^{\infty} Q(\sqrt{2 \gamma}) p_{\gamma_{2}}(\gamma) \mathrm{d} \gamma \\
& =\left[\frac{1}{2}\left(1-\mu_{2}\right)\right]^{2 N M} \sum_{i=0}^{2 N M-1}\left(\begin{array}{c}
2 N M-1+i \\
i
\end{array}\right)\left[\frac{1}{2}\left(1+\mu_{2}\right)\right]^{i}
\end{aligned}
$$

where

$$
\mu_{2} \triangleq \sqrt{\frac{E_{b} / N_{0} / N}{1+E_{b} / N_{0} / N}}
$$

It should be noted that the correlative detector has an $M$-fold (spatial) diversity, whereas the time-diversity detector has an $M N$-fold (spatial and temporal) diversity.

For the iterative detection, since the conditional SNR $\gamma$ is the summation of the independent conditional SNRs $\gamma_{1}$ and $\gamma_{2}$, the pdf of $\gamma$ is the convolution of the gamma pdfs of $\gamma_{1}$ and $\gamma_{2}$. This is written as follows:

$$
\begin{aligned}
p_{\gamma}(\gamma) & =p_{\gamma_{1}}(\gamma) \otimes p_{\gamma_{2}}(\gamma) \\
& =\frac{\left(\gamma^{M-1} \mathrm{e}^{-\frac{\gamma}{E_{b} / N_{0}}}\right) \otimes\left(\gamma^{N M-1} \mathrm{e}^{-\frac{\gamma}{E_{b} / N_{0} / N}}\right)}{(M-1) !(N M-1) !\left(E_{b} / N_{0}\right)^{M}\left(E_{b} / N_{0} / N\right)^{N M}}, \quad \gamma \geq 0 .
\end{aligned}
$$

The BER expression can then be obtained by averaging $Q(\sqrt{2 \gamma})$ over the conditional pdf of $\gamma$.

The complexity of such a calculation can be obviated with the observation that the pdf $p_{\gamma_{2}}(\gamma)$ approaches a Dirac delta function as $N$ becomes large.

This stems from the fact that the gamma distribution approaches a Gaussian distribution if the degree of freedom, here 2NM, is large [18]. Furthermore, the Gaussian distribution tends toward a Dirac delta function as its variance, here $2 N M\left(E_{b} / N_{0} / N\right)^{2}=2 M\left(E_{b} / N_{0}\right)^{2} / N$, becomes very small [18]. It follows that if the spreading factor $N$ is sufficiently large, the distribution of $p_{\gamma_{2}}(\gamma)$ can be approximated by an impulse located at the mean value of $\gamma_{2}$, which is given as $2 N M\left(E_{b} / N_{0} / N\right)=2 M\left(E_{b} /\right.$ $\left.N_{0}\right)$. In other words, $p_{\gamma_{2}}(\gamma)$ is approximatively equal to $\delta\left(\gamma-2 M E_{b} / N_{0}\right)$. 
So as $N$ becomes large, the pdf of $\gamma_{2}$ approaches an impulse and the pdf of $\gamma$ can be obtained as the shifted pdf of $\gamma_{1}$ :

$$
\begin{aligned}
p_{\gamma}(\gamma) & \approx p_{\gamma_{1}}(\gamma) \otimes \delta\left(\gamma-2 M E_{b} / N_{0}\right) \\
& = \begin{cases}\frac{\gamma-2 M E_{b} / N_{0}}{E_{b} / N_{0}} & , \gamma \geq 2 M \frac{E_{b}}{N_{0}} \\
\frac{\left(\gamma-2 M E_{b} / N_{0}\right)^{2 M-1} e}{(2 M-1) !\left(E_{b} / N_{0}\right)^{2 M}} & \text { else }\end{cases}
\end{aligned}
$$

The BER of the iterative detector can be calculated as

$$
\begin{aligned}
P_{e} & =\int_{0}^{\infty} Q(\sqrt{2 \gamma}) p_{\gamma}(\gamma) \mathrm{d} \gamma \\
\approx & \int_{2 M E_{b} / N_{0}}^{\infty} Q(\sqrt{2 \gamma}) \frac{\left(\gamma-2 M E_{b} / N_{0}\right)^{2 M-1} e^{-\frac{\gamma-2 M E_{F} / N_{0}}{k_{b} N_{0}}}}{(2 M-1) !\left(E_{b} / N_{0}\right)^{2 M}} \mathrm{~d} \gamma \\
= & Q\left(\sqrt{4 M \frac{E_{b}}{N_{0}}}\right)- \\
& \sqrt{\frac{\mu_{1}}{2 \pi}} \frac{2 M}{\sum_{k=0}^{2 M-1}} \frac{N_{0}^{k}}{E_{b}^{k} k !} \sum_{l=0}^{k}\left(\begin{array}{l}
k \\
l
\end{array}\right)\left(\mu_{1}\right)^{k-1}\left(-2 M \frac{E_{b}}{N_{0}}\right)^{l} \Gamma\left(k-l+\frac{1}{2}, 2 M\left(1+\frac{E_{b}}{N_{0}}\right)\right)
\end{aligned}
$$

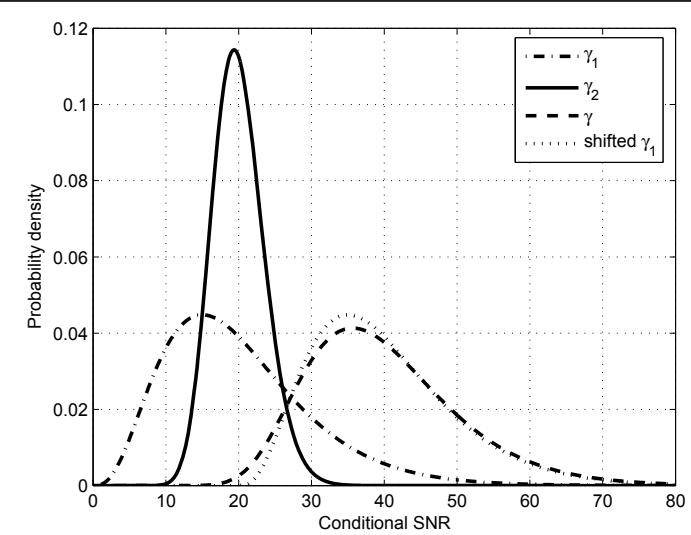

(a) $2 \times 2, \mathrm{~N}=8, \mathrm{SNR}=10 \mathrm{~dB}$

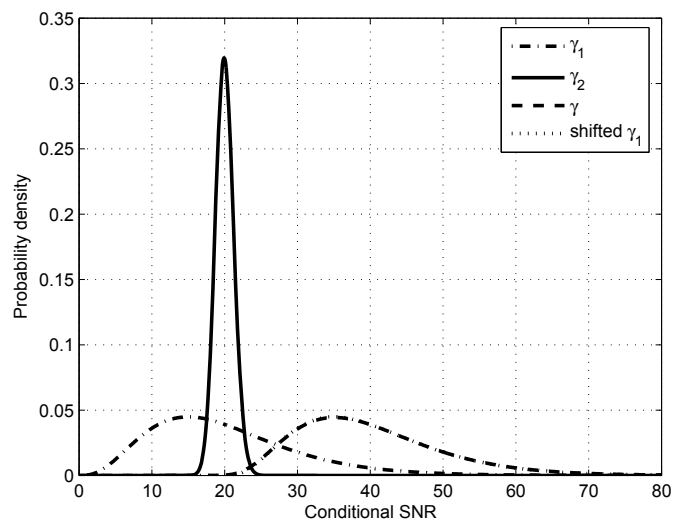

(c) $2 \times 2, \mathrm{~N}=64, \mathrm{SNR}=10 \mathrm{~dB}$
Appendix A presents the derivation of this result.

We note that the impulse approximation of the pdf yields a lower bound for the BER, because it can only be approached when the spreading factor $N$ is sufficiently large.

\section{Analytical and simulation results}

4.1 Probabilistic models

In this section, we present the distribution of the conditional SNRs and discuss the delta approximation related to the iterative detection. Figure 3 shows the probability density functions of the conditional SNRs of the correlation detection $\gamma_{1}$, diversity detection $\gamma_{2}$, and iterative detection $\gamma$, for different scenarios. Each sub-figure includes an extra curve that shows the distribution of $\gamma_{1}$ that has been right-shifted by $2 M \frac{E_{b}}{N_{0}}$. The coordinates for different sub-figures have been adjusted in order to illustrate the distributions in detail. As analyzed in

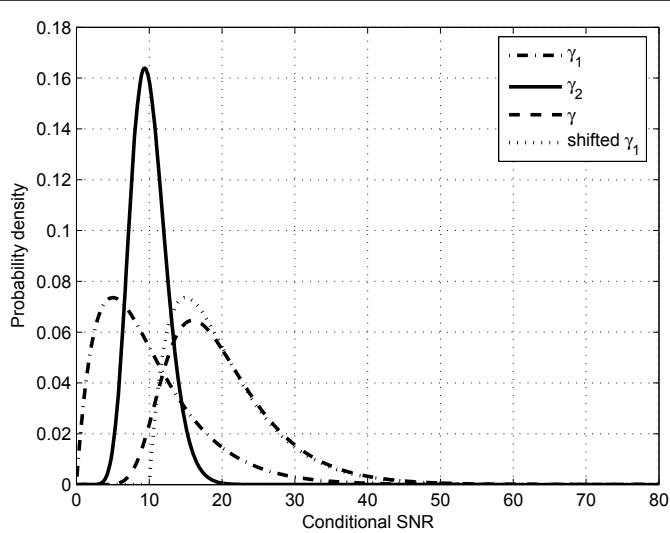

(b) $2 \times 1, \mathrm{~N}=8, \mathrm{SNR}=10 \mathrm{~dB}$

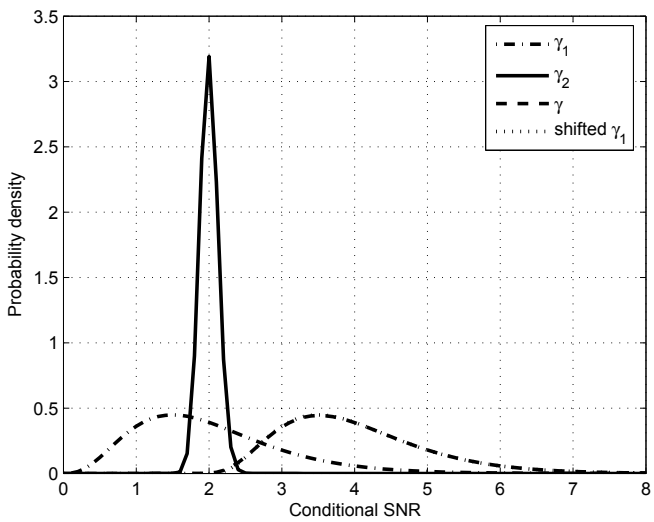

(d) $2 \times 2, \mathrm{~N}=64, \mathrm{SNR}=0 \mathrm{~dB}$

Figure 3 Distribution of the conditional SNRs 
Section 3, the mean and variance of the conditional SNR of the diversity detection $\gamma_{2}$ are $2 M \frac{E_{b}}{N_{0}}$ and $\frac{2 M}{N}\left(\frac{E_{b}}{N_{0}}\right)^{2}$, respectively. This means that while the number of receive antennas and the SNR affect both position and shape of the "pulse," the spreading length only influences its shape. Figure $3 \mathrm{a}$ and $3 \mathrm{~b}$ show that the position and the shape of the pulse vary with the number of antennas. In both cases, the pulse is not very narrow due to the small value of $N=8$, resulting in a discernable difference between the shifted version of the distribution of $\gamma_{1}$ (dotted curve) and the distribution of $\gamma$ (dashed curve). The "pulse" looks sharper in Figure 3c since $N=64$ is much larger. Because the area under the pdf is fixed, this sharper pulse approximates a delta function such that there is negligible difference between the shifted distribution of $\gamma_{1}$ and the distribution of $\gamma$. Also, Figure $3 \mathrm{c}$ and $3 \mathrm{~d}$ shows that the delta approximation is not very sensitive to SNR, although it is more accurate with lower SNR.

\subsection{Performance comparisons}

Next, we present the BER performance of MIMO-SESS and compared with a conventional MIMO, PN-coded spread spectrum (MIMO-PNSS) system. The channel between each transmit and receive antenna pair is assumed to be Rayleigh fading that remains constant over $T_{b}$ but is independent from bit to bit $[6,16]$. In addition, the channels for different transmit/receive antenna pair are independent. The length of the spreading sequence is set to $N=64$ unless noted otherwise. For each scenario and bit signal-to-noise ratio (SNR), 100 runs of 100,000 bits are simulated to evaluate the average bit error rate (BER).

Figure 4 compares the BER of MIMO-SESS with correlation detection to a MIMO-PNSS system. It is

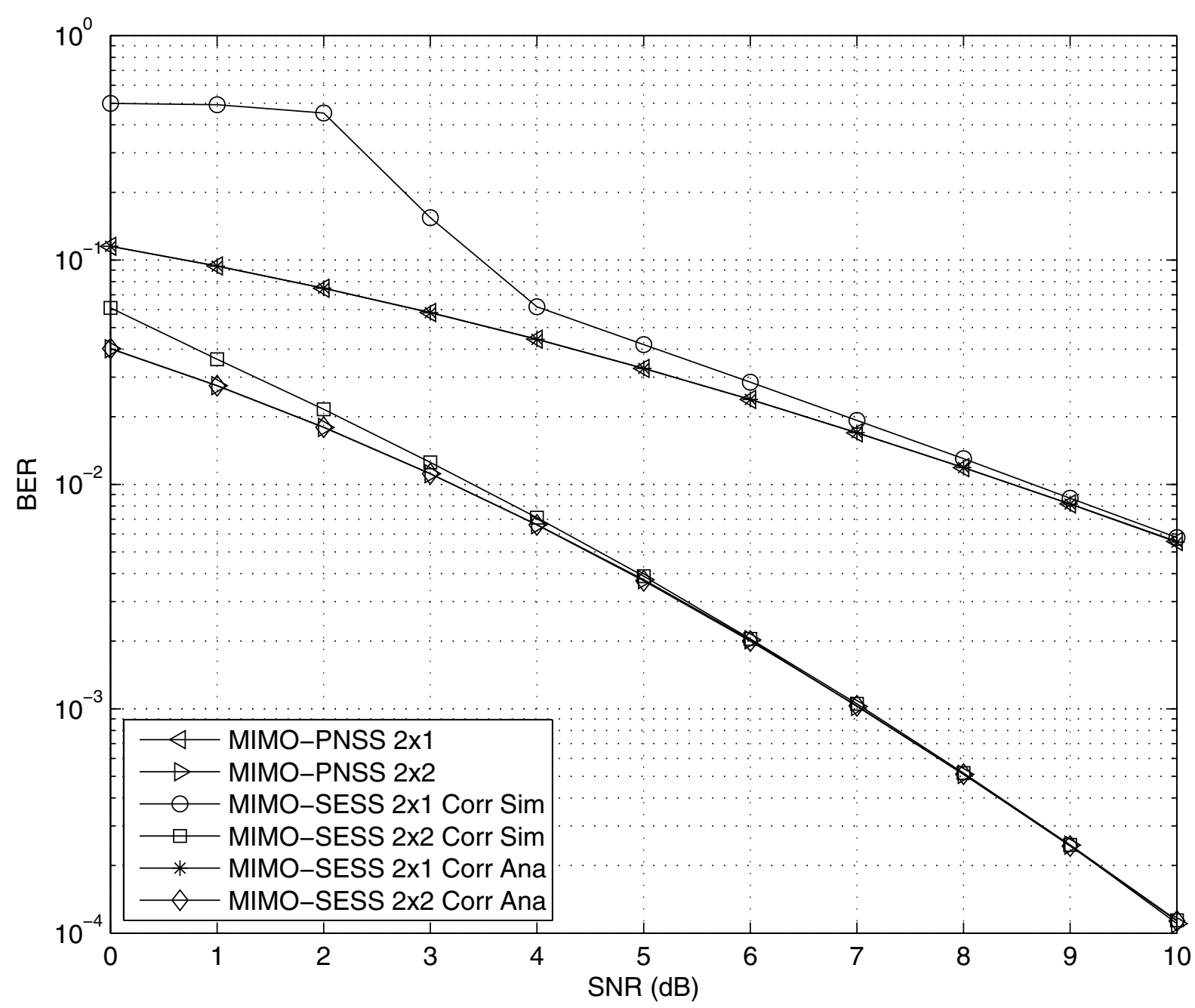

Figure 4 Comparison between MIMO-PNSS and MIMO-SESS with correlation detection, $N=64$ 
observed that without iterative detection, there is no time diversity gain, and thus, the performance with or without self-encoding is the same as expected under high SNR. The performance degradation of MIMOSESS at low SNR is due to error propagation [1]. In particular, Figure 4 shows that the effect of error propagation is quite severe for a BER greater than $10 \%$. The effect of error propagation can be efficiently alleviated with more antennas, as shown by the example $2 \times 2$ scenario, since larger spatial diversity reduces BER to below $10 \%$ even at low SNR. The plots in Figure 4 show excellent agreement between the analytical and simulation results at high SNR, where error propagation becomes insignificant.

The performance of MIMO-SESS with time-diversity detection is shown in Figure 5. The plots show that the proposed system significantly outperforms conventional MIMO-PNSS systems. As an example, there is about 4.5
$\mathrm{dB}$ gain for the $2 \times 2$ configuration at $10^{-4} \mathrm{BER}$. This performance improvement can be attributed to the $N$ fold time diversity introduced by self encoding. Due to the significantly improved BER, error propagation is effectively mitigated as shown by the $2 \times 2$ scenario. Moveover, the agreement with the simulation results verifies that the analysis has been justified in ignoring error propagation.

Figure 6 compares the BER with the iterative detector. Again, there is excellent agreement between the analytical and simulation results at sufficiently high SNR. The performance gain over conventional MIMO-PNSS systems is almost $7 \mathrm{~dB}$ at $10^{-4} \mathrm{BER}$ for the $2 \times 2$ configuration. The excess gain beyond the expected $3 \mathrm{~dB}$ SNR improvement can be attributed to the diversity gain from the time-diversity detection. In fact, the plot shows that a $2 \times 2$ MIMO-SESS would require an SNR of only $3 \mathrm{~dB}$ to achieve a $10^{-4} \mathrm{BER}$. The results verify the

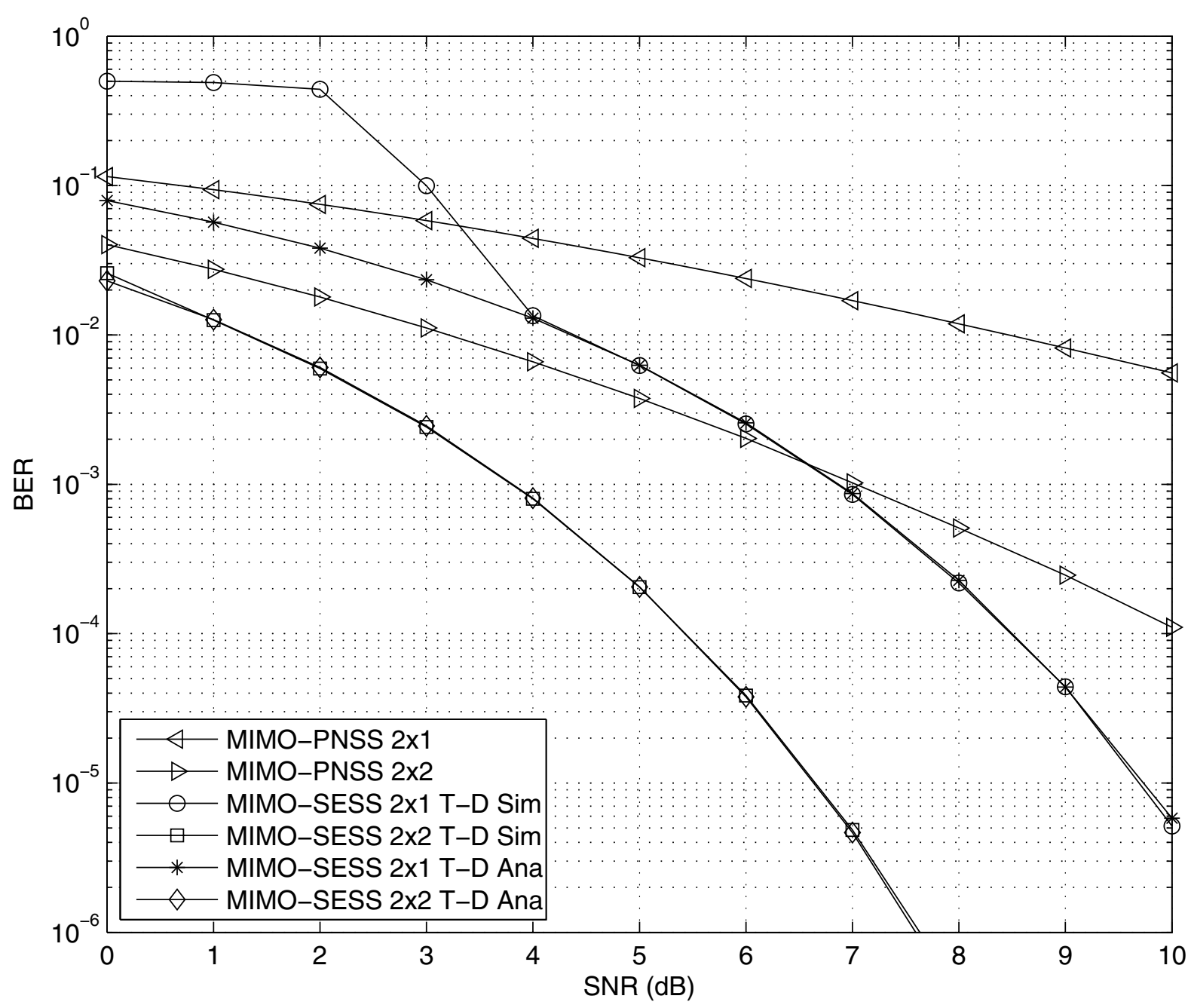

Figure 5 Comparison between MIMO-PNSS and MIMO-SESS with diversity detection, $N=64$ 
veracity of the performance analysis and demonstrate that MIMO-SESS can completely mitigate the effect of Rayleigh fading. The difference between the numerical and simulation results at low SNR is due to error propagation. This can be easily demonstrated in simulation by feeding the original (transmitted) bits into the delay registers at the receiver. The results of this simulation are compared with the numerical calculations in Figure 7. The excellent agreement between the simulation and numerical results demonstrates the validity of the statistical models and approximations.

Figure 8 shows the numerical results of MIMO-SESS with the iterative detection for different spreading lengths. For both $2 \times 1$ and $2 \times 2$ cases, the performance approaches the lower bound when the spreading length increases. It should be noted that the lower bound is very tight for $N \geq 64$. Also, the lower bound is tighter for the $2 \times 1$ case and at lower SNR. These results are consistent with the discussion in Section 3. It can be seen by comparing the lower bound and the performance of BPSK in AWGN that $2 \times 2$ MIMO-SESS can provide about $5.2 \mathrm{~dB}$ gain over BPSK at $10^{-4}$ BER. This significant performance improvement is clearly due to the combined temporal and spatial diversities associated with MIMO-SESS design.

\subsection{Remarks}

The effect of error propagation is largely confined to high BERs $\left(>10^{-2}\right)$ as the bit errors are fed back to the de-spreading chips and propagate through the receiver delay registers. Thus, the benefit of MIMO reducing the effect of error propagation occurs in the low SNR regions (primarily through the $3 \mathrm{~dB}$ array gain) as shown by the simulation results. However, the performance gaps between error propagation and "no" error propagation (true bits) remain for low BER. The

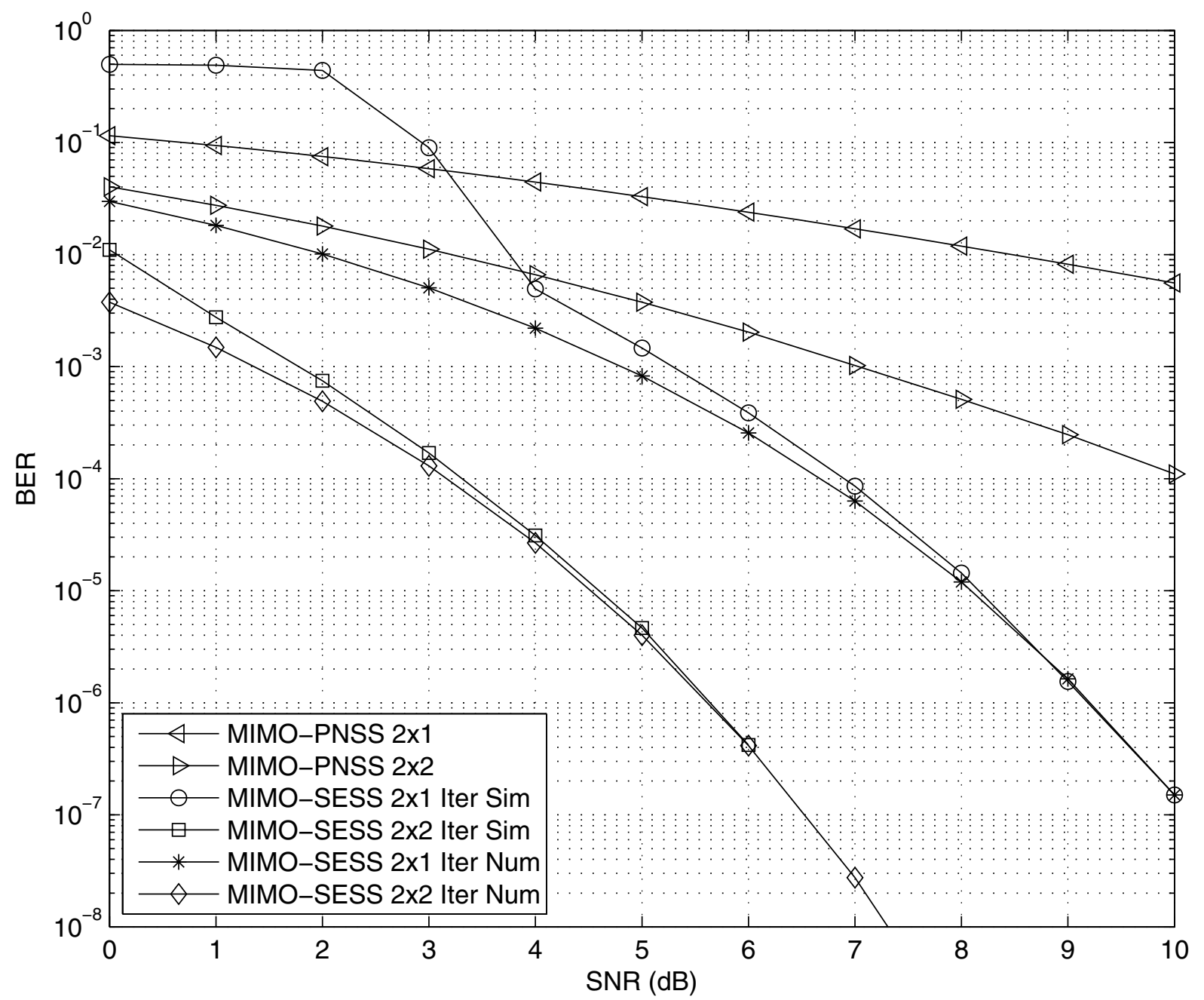

Figure 6 Comparison between MIMO-PNSS and MIMO-SESS with iterative detection, $N=64$. 


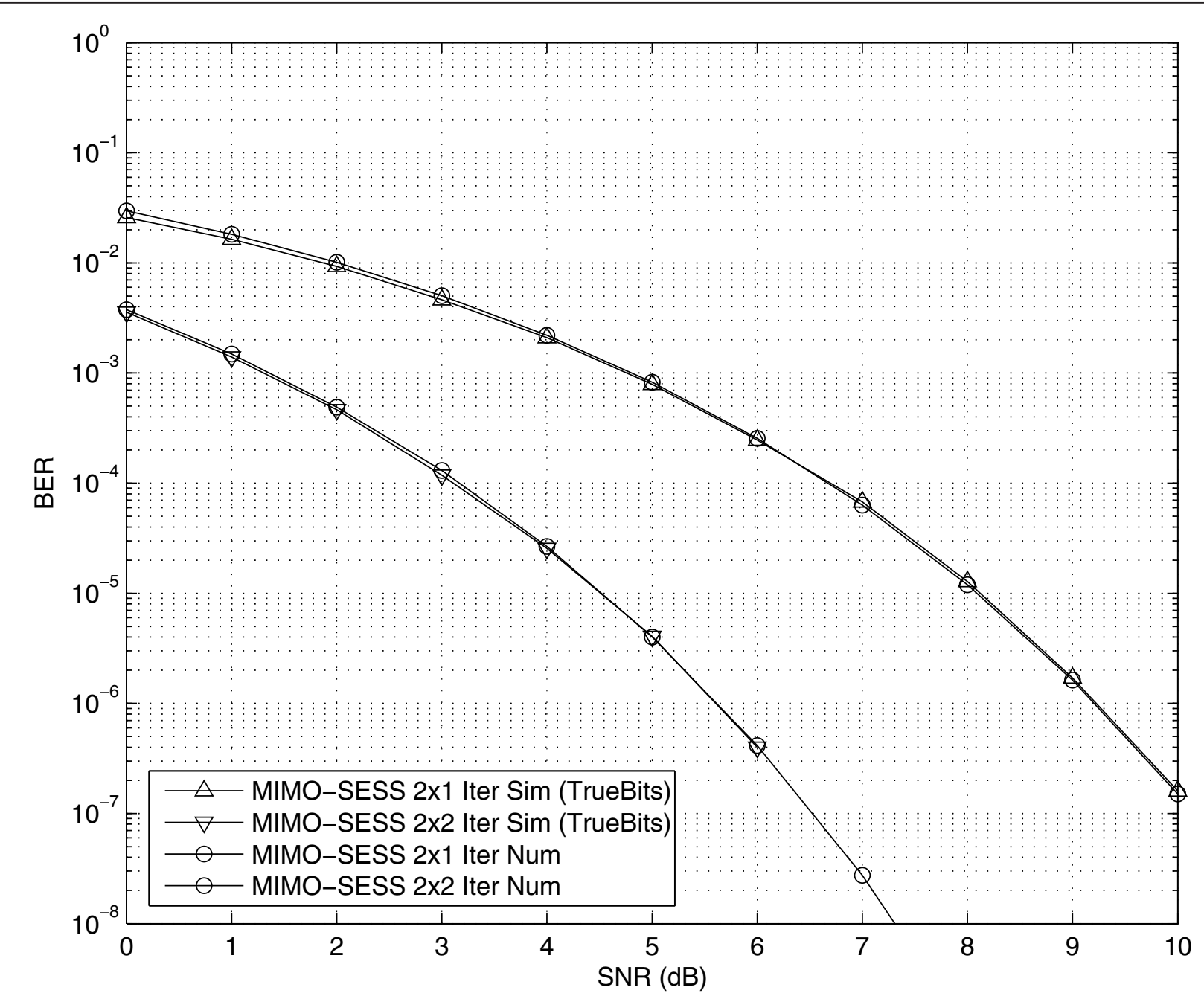

Figure 7 Comparison between numerical results and simulation results without error propagation, $N=64$

comparison with the analytical results also showed that the discrepancies are primarily in high BER, low SNR regions. This suggests that in practical systems where the BER is sufficiently low, the effect of error propagation and hence its mitigation with MIMO scheme are rather minimal and relatively insignificant. For example at $10^{-3} \mathrm{BER}$, the performance gap is negligible for correlation and time diversity detectors, and amounts to less than $0.5 \mathrm{~dB}$ for the iterative detector.

The results showed that MIMO scheme provides spatial diversity under fading that is most beneficial to correlation detection (which has no diversity). Time diversity detection on the other hand can achieve $\mathrm{N}$-fold diversity that completely mitigates the effect of fading; so there is very little spatial diversity benefit from MIMO scheme especially under high SNR. Since iterative detection combines time diversity and correlation detection, it also benefit from spatial diversity but to a lesser extent. The advantage of the combination of MIMO and SESS therefore is to exploit the array gain to mitigate error propagation at low SNR and the spatial diversity to improve the overall BER.

\section{Conclusion}

In this paper, we presented the analytical study of the BER performance of MIMO-SESS over Rayleigh fading channels. We derived the closed-form BER expressions for the correlation detector and time diversity detector, and obtained a lower bound for the iterative detector. The veracity of the analysis has been confirmed with the numerical calculations based on the analytical expressions, which have demonstrated excellent agreement with the simulation results. The performance analysis has shown that MIMO-SESS offers almost $7 \mathrm{~dB}$ of gain at a $10^{-4}$ BER compared with a $2 \times 2$ MIMO PN-coded spread spectrum system. Furthermore, the system 


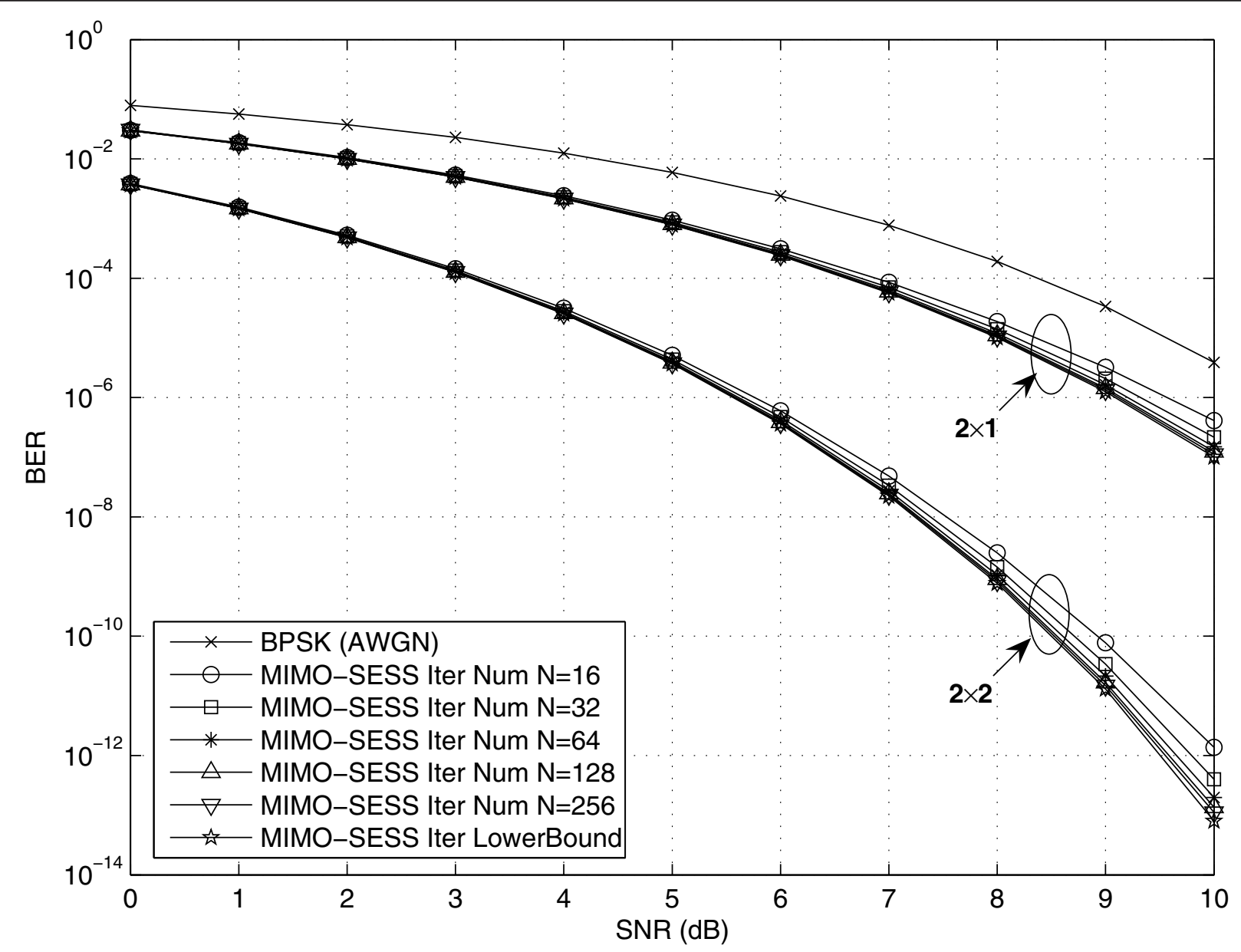

Figure 8 Performance of the proposed MIMO-SESS system with various $N$ values.

requires only about $3 \mathrm{~dB}$ SNR to achieve a BER of $10^{-4}$. This demonstrates that MIMO-SESS can provide a very effective means to exploit both spatial and temporal diversities in order to achieve robust performance in wireless environments.

Although we have examined an Alamouti schemebased MIMO system with two transmitting antennas, the MIMO-SESS analysis that has been developed here can be easily applied to other orthogonal space-time block coded MIMO systems with any number of antennas at transmitter side. Additional study is being undertaken to extend this work toward multi-code MIMOSESS that can improve the transmission rate, system throughput, and spectral efficiency.

\section{Appendix}

A Derivation of Equation 36

This appendix provides a detailed derivation of Equation (36). Define $a=2 M E_{b} / N_{0}, b=E_{b} / N_{0}$, and $L=2 M$, then the BER of the iterative detector can be written as

$$
\begin{aligned}
P_{e} & \approx \int_{a}^{\infty} Q(\sqrt{2 \gamma}) \frac{(\gamma-a)^{L-1} \mathrm{e}^{-\frac{\gamma-a}{b}}}{(L-1) ! b^{L}} \mathrm{~d} \gamma \\
& =\int_{0}^{\infty} Q(\sqrt{2(x+a)}) \frac{x^{L-1} \mathrm{e}^{-\frac{x}{b}}}{(L-1) ! b^{L}} \mathrm{~d} x \\
& =\int_{0}^{\infty} \frac{1}{\sqrt{2 \pi}} \int_{\sqrt{2(x+a)}}^{\infty} \mathrm{e}^{-\frac{u^{2}}{2}} d u \frac{x^{L-1} \mathrm{e}^{-\frac{x}{b}}}{(L-1) ! b^{L}} \mathrm{~d} x
\end{aligned}
$$

By changing the order of the integrals, (37) becomes

$$
P_{e} \approx \frac{1}{\sqrt{2 \pi}} \frac{1}{(L-1) ! b^{L}} \int_{\sqrt{2 a}}^{\infty} e^{-\frac{u^{2}}{2}} P_{1} \mathrm{~d} u
$$

where

$$
P_{1}=\int_{0}^{\frac{u^{2}}{2}-a} x^{L-1} \mathrm{e}^{-\frac{x}{b}} \mathrm{~d} x
$$


Let $z=\frac{x}{b}$, then

$$
P_{1}=b^{L} \int_{0}^{\frac{u^{2}}{2 b}-\frac{a}{b}} z^{L-1} \mathrm{e}^{-z} \mathrm{~d} x
$$

Using the indefinite integral equation given in [[19], Eq. 7.4.322], $P_{1}$ can evaluated as

$$
P_{1}=-b^{L} \mathrm{e}^{-\frac{u^{2}}{2+}+\frac{a}{b}} \sum_{k=1}^{L-1} \frac{(L-1) !}{k !}\left(\frac{u^{2}}{2 b}-\frac{a}{b}\right)^{k}-b^{L}(L-1) !\left(1-\mathrm{e}^{-\frac{u^{2}}{2 b^{2}}+\frac{a}{b}}\right)
$$

Substituting (41) into (38) to determine $P_{e}$ :

$$
\begin{aligned}
P_{e} & \approx \int_{\sqrt{2 a}}^{\infty} \frac{1}{\sqrt{2 \pi}} \mathrm{e}^{-\frac{u^{2}}{2}} \mathrm{~d} u-\frac{1}{\sqrt{2 \pi}} \mathrm{e}^{\frac{a}{b}} \int_{\sqrt{2 a}}^{\infty} \mathrm{e}^{-\frac{u^{2}}{2}\left(\frac{1+b}{b}\right)} \mathrm{d} u \\
& -\frac{1}{\sqrt{2 \pi}} \mathrm{e}^{\frac{a}{b}} \sum_{k=1}^{L-1} \frac{1}{k !}\left(\frac{1}{2 b}\right)^{k} \int_{\sqrt{2 a}}^{\infty} \mathrm{e}^{-\frac{u^{2}}{2}\left(\frac{1+b}{b}\right)}\left(u^{2}-2 a\right)^{k} \mathrm{~d} u \\
& =Q(\sqrt{2 a})-\frac{1}{\sqrt{2 \pi}} \mathrm{e}^{\frac{a}{b}} \sum_{k=0}^{L-1} \frac{1}{k !}\left(\frac{1}{2 b}\right)^{k} P_{2}
\end{aligned}
$$

where

$$
P_{2}=\int_{\sqrt{2 a}}^{\infty} \mathrm{e}^{-\frac{u^{2}}{2}\left(\frac{1+b}{b}\right)}\left(u^{2}-2 a\right)^{k} \mathrm{~d} u
$$

This can be further transformed with the substitution

$$
\begin{aligned}
P_{2} & =2^{k-1} \sqrt{\frac{2 b}{1+b}} \int_{\frac{a(1+b)}{b}}^{\infty} \mathrm{e}^{-t} t^{-\frac{1}{2}}\left(\frac{b}{1+b} t-a\right)^{k} \mathrm{~d} t \\
& =2^{k-1} \sqrt{\frac{2 b}{1+b}} \int^{\frac{a(1+b)}{b}} \mathrm{e}^{-t} t^{-\frac{1}{2}} \sum_{l=0}^{k}\left(\begin{array}{l}
k \\
l
\end{array}\right)\left(\frac{b}{1+b} t\right)^{k-l}(-a)^{l} \mathrm{~d} t \quad: \\
& =2^{k-1} \sqrt{\frac{2 b}{1+b}} \sum_{l=0}^{k}\left(\begin{array}{l}
k \\
l
\end{array}\right)\left(\frac{b}{1+b}\right)^{k-l}(-a)^{l} \Gamma\left(k-l+\frac{1}{2}, \frac{a(1+b)}{b}\right) \\
P_{2} & =2^{k-1} \sqrt{\frac{2 b}{1+b}} \int_{\frac{a(1+b)}{b}}^{\infty} \mathrm{e}^{-t} t^{-\frac{1}{2}}\left(\frac{b}{1+b} t-a\right)^{k} \mathrm{~d} t \\
& =2^{k-1} \sqrt{\frac{2 b}{1+b}} \int_{\frac{a(1+b)}{b}}^{\infty} \mathrm{e}^{-t} t^{-\frac{1}{2}} \sum_{l=0}^{k}\left(\begin{array}{l}
k \\
l
\end{array}\right)\left(\frac{b}{1+b} t^{k}\right)^{k-l}(-a)^{l} \mathrm{~d} t \\
& =2^{k-1} \sqrt{\frac{2 b}{1+b}} \sum_{l=0}^{k}\left(\begin{array}{l}
k \\
l
\end{array}\right)\left(\frac{b}{1+b}\right)^{k-l}(-a)^{l} \Gamma\left(k-l+\frac{1}{2}, \frac{a(1+b)}{b}\right)
\end{aligned}
$$

where $\Gamma(a, x)$ denotes the incomplete gamma function with shape $a$ and scale $x$ [[20], Eq. 6.5.3]. Substituting (44) into (42), we have:

$$
\begin{aligned}
P_{e} \approx Q & (\sqrt{2 a})-\frac{1}{\sqrt{2 \pi}} e^{\frac{a}{b}} \sqrt{\frac{b}{1+b}} \\
& \times \sum_{k=0}^{L-1} \frac{1}{b^{k} k !} \sum_{l=0}^{k}\left(\begin{array}{l}
k \\
l
\end{array}\right)\left(\frac{b}{1+b}\right)^{k-l}(-a)^{l} \Gamma\left(k-l+\frac{1}{2}, \frac{a(1+b)}{b}\right)
\end{aligned}
$$

The final expression for $P_{e}$ in (36) can be obtained with the defined values for $a, b$, and $L$ :

$$
\begin{aligned}
& P_{e} \approx Q\left(\sqrt{4 M \frac{E_{b}}{N_{0}}}\right)- \\
& \sqrt{\frac{\mu_{1}}{2 \pi} e^{2 M}} \sum_{k=0}^{2 M-1} \frac{N_{0}^{k} k}{E_{b}^{k} !} \sum_{l=0}^{k}\left(\begin{array}{l}
k \\
l
\end{array}\right)\left(\mu_{1}\right)^{k-1}\left(-2 M \frac{E_{b}}{N_{0}}\right)^{l} \Gamma\left(k-l+\frac{1}{2}, 2 M\left(1+\frac{E_{b}}{N_{0}}\right)\right)
\end{aligned}
$$

where $\mu_{1}$ is given by (30).

\section{Acknowledgements}

This work was funded in part by contract award FA9550-08-1-0393 from the US Air Force Office of Scientific Research. The authors wish to thank Dr. J. Sjogren for his support of this study. This paper was presented in part at IEEE International Conference on Communications (ICC), Cape Town, South Africa, May 2010, and at ISITA/ISSSTA2010, Taichung, Taiwan, Oct. 2010.

\section{Competing interests}

The authors declare that they have no competing interests.

Received: 28 April 2011 Accepted: 27 October 2011 Published: 27 October 2011

\section{References}

1. L Nguyen, Self-encoded spread spectrum communications, in Proceedings of Military Communications Conference Proceedings (MILCOM 1999). 1 182-186 (1999)

2. L Nguyen, Self-encoded spread spectrum and multiple access communications, in Proceedings of IEEE Sixth International Symposium on Spread Spectrum Techniques and Applications, vol. 2. (Parsippany, NJ, 2000), pp. 394-398

3. W Jang, L Nguyen, Capacity analysis of $m$-user self-encoded multiple access system in AWGN channels, in Proceedings of IEEE Sixth International Symposium on Spread Spectrum Techniques and Applications. 1, 216-220 (2000)

4. Y Kong, L Nguyen, W Jang, Self-encoded spread spectrum modulation with differential encoding, in Proceedings of IEEE Seventh International Symposium on Spread Spectrum Techniques and Applications. 2, 471-474 (2002)

5. WM Jang, L Nguyen, M Hempel, Self-encoded spread spectrum and turbo coding. J Commun Netw. 6(1), 9-18 (2004)

6. YS Kim, W Jang, Y Kong, L Nguyen, Chip-interleaved self-encoded multiple access with iterative detection in fading channels. J Commun Netw. 9(1), 50-55 (2007)

7. K Hua, L Nguyen, W Jang, Synchronisation of self-encoded spread spectrum system. Electron Lett. 44(12), 749-751 (2008). doi:10.1049/el:20083457

8. S Ma, L Nguyen, WM Jang, Y Yang, Multiple-input multiple-output selfencoded spread spectrum system with iterative detection, in Proceedings of IEEE International Conference on Communications (ICC'10), (Cape Town, South Africa, 2010)

9. A Paulraj, R Nabar, D Gore, Introduction to Space-Time Wireless Communications, (Cambridge University Press, Cambridge, UK, 2003)

10. D Gesbert, M Shafi, D Shan Shiu, PJ Smith, A Naguib, From theory to practice: an overview of MIMO space-time coded wireless systems. IEEE J Sel Areas Commun. 21(3), 281-302 (2003). doi:10.1109/JSAC.2003.809458

11. AJ Paulraj, DA Gore, RU Nabar, H Bölcskei, An overview of MIMO communications-a key to gigabit wireless. Proc IEEE. 92(2), 198-218 (2004). doi:10.1109/JPROC.2003.821915

12. IEEE, IEEE Standard for Local and Metropolitan Area Networks, Part 16: Air Interface for Fixed Broadband Wireless Access Systems. IEEE Std 802.16-2004 (2004)

13. IEEE, IEEE Candidate Standard 802.11n: Wireless LAN Medium Access Control (MAC) and Physical Layer (PHY) Specifications. (2004)

14. 3GPP Technical Specification Group Radio Access Network, Overall description of E-UTRA/E-UTRAN; stage 2. Tech Rep TR 36.300 (2007)

15. S Ma, L Nguyen, WM Jang, Y Yang, MIMO self-encoded spread spectrum with iterative detection over rayleigh fading channels. Hindawi J Electr Comput Eng. 2010, 1-9 (2010). Article ID 492079

16. JG Proakis, Digital Communications, 4th edn. (McGraw-Hill, New York, 1989)

17. S Alamouti, A simple transmit diversity technique for wireless communicaiton. IEEE J Sel Areas Commun. 16(8), 1451-1458 (1998). doi:10.1109/49.730453 
18. PG Hoel, SC Port, CJ Stone, Introduction to Probability Theory (Houghton Mifflin, Boston, 1971)

19. L Rade, B Westergren, Mathematics Handbook for Science and Engineering, (Birkhauser Inc., Boston, Cambridge, MA, USA, 1995)

20. M Abramowitz, IA Stegun, Handbook of Mathematical Functions With Formulas, Graphs, and Mathematical Tables (Dover, New York, 1965)

doi:10.1186/1687-1499-2011-145

Cite this article as: Ma et al.: Performance analysis of MIMO-SESS with

Alamouti scheme over Rayleigh fading channels. EURASIP Journal on

Wireless Communications and Networking 2011 2011:145.

Submit your manuscript to a SpringerOpen ${ }^{\odot}$ journal and benefit from:

- Convenient online submission

- Rigorous peer review

- Immediate publication on acceptance

- Open access: articles freely available online

- High visibility within the field

- Retaining the copyright to your article

Submit your next manuscript at $\gg$ springeropen.com 